\title{
Flow visualization of free convection in a vertical cylinder of water in the vicinity of the density maximum
}

\author{
M.F. Cawley *, P. McBride \\ Department of Experimental Physics, National University of Ireland, Maynooth, County Kildare, Ireland \\ Received 24 February 2003; received in revised form 16 September 2003
}

\begin{abstract}
Free convection in a vertical cylinder of water is studied in the vicinity of the density maximum at $4{ }^{\circ} \mathrm{C}$. Results are presented both from cooling curve measurements (using a series of thermistors along the central axis of the cylinder) and from flow visualization experiments (using particle image velocimetry), and a comparison is made with simulations. The reversal of the overall flow direction as the temperature crosses the maximum density region gives rise to a plateau feature in the cooling curve, which may be explained by the formation of a rising toroidal structure along the column boundary. This feature effectively diverts flow away from the inner region of the cylinder, resulting in a temporary cessation of convective cooling within this region.
\end{abstract}

(c) 2003 Elsevier Ltd. All rights reserved.

\section{Introduction}

Fluid density usually changes as a function of temperature in a reasonably linear manner. A notable exception is liquid water. Pure water at a pressure of one atmosphere has a maximum density of $999.9720 \mathrm{~kg} \mathrm{~m}^{-3}$ at $4{ }^{\circ} \mathrm{C}[1]$. Above this temperature the density of water decreases as the temperature is increased in a manner similar to other fluids. For temperatures below $4{ }^{\circ} \mathrm{C}$, however, the trend is reversed: density increases with increased temperature, giving rise to a maximum density at the $4{ }^{\circ} \mathrm{C}$ point. The density profile for pure water at $101 \mathrm{kPa}$ (one atmosphere), in the vicinity of its density maximum, is shown in Fig. 1. There is a $0.013 \%$ increase in density between 0 and $4{ }^{\circ} \mathrm{C}$, and a decrease in density of a similar magnitude between 4 and $8{ }^{\circ} \mathrm{C}$; the magnitude of the density anomaly in the liquid state is therefore small compared to the anomalous contraction by $8.3 \%$ in going from solid to liquid water (an anomaly magnitude ratio of 640:1).

One of the earliest detailed studies of the liquid water density anomaly was carried out by Thomas Charles

\footnotetext{
*Corresponding author. Fax: +353-1-7083313.

E-mail address: mcawley@may.ie (M.F. Cawley).
}

Hope [2]. Through a series of experiments, he proposed that water was at its most dense at a temperature between 4.2 and $4.4{ }^{\circ} \mathrm{C}$ (Hope quoted his results using the Fahrenheit scale; he concluded that the density maximum lay between 39.5 and $40{ }^{\circ} \mathrm{F}$ ). To circumvent objections, prevalent at that time, that the density anomaly was due to expansion or contraction of the vessel in which the water sample was contained, the experiments devised by Hope did not rely on measurement of volume (and hence density) changes. Instead, Hope's result relied primarily on measuring the temperature of the dense water which settled at the bottom of a cooled vertical cylinder. By noting that a temperature inversion occurred, with warmer water settling below cooler-less dense-water, Hope concluded that the water density anomaly existed independent of any complications due to changes in container volume.

The effects of the density anomaly may also be detected in modifications to expected free convective flow. Being driven by buoyancy forces, such free convection flows are sensitive to small changes in the density of bulk regions within the fluid. A density reversal will be particularly disruptive to buoyancy-driven flow, as it will ultimately result in the reversal of the flow direction (assuming the effect persists for a sufficient duration). In this work we present results from a visualization study 


\begin{tabular}{|llll|}
\hline \multicolumn{2}{|l|}{ Nomenclature } & & \\
$\mathbf{g}$ & gravitational acceleration vector & $(u, v)$ & velocity components \\
$h$ & height of cavity & $\mathbf{v}$ & velocity vector \\
$p$ & pressure & $w$ & width of cavity \\
$t$ & time & Greek & symbols \\
$T$ & temperature & $\alpha$ & coefficient of thermal diffusivity \\
$T_{0}$ & reference temperature & $\beta$ & coefficient of thermal expansion \\
$T_{\mathrm{c}}$ & coolant temperature, cold & $\mu$ & dynamic viscosity \\
$T_{\mathrm{h}}$ & coolant temperature, warm & $\rho$ & density of liquid \\
$T_{\mathrm{p}, \mathrm{c}}$ & plateau temperature, cooling curve & $\rho_{0}$ & reference density \\
$T_{\mathrm{p}, \mathrm{h}}$ & plateau temperature, warming curve & & \\
$T_{\rho \max }$ & temperature of density maximum & & \\
\hline
\end{tabular}

of water undergoing free convection in a temperature region bracketing the density maximum temperature. We follow the approach of Anselmi et al. [3]: water at $8{ }^{\circ} \mathrm{C}$ in a vertical cylinder is surrounded by fluid maintained at $-2{ }^{\circ} \mathrm{C}$, and the convective flow patterns are studied as the test sample cools through the $4{ }^{\circ} \mathrm{C}$ region (the subzero cooling temperature permits testing over a wider temperature range bracketing the temperature of density maximum; the anomalous density behaviour of water persists into the supercooled region). In de Paz et al. [4], Anselmi et al. [3], and Sonnino and de Paz [5], experimental observations were made of the temperatures at various points along the central axis of the cylinder. We repeat these measurements, and extend the study to include two-dimensional visualization of the flow patterns using particle image velocimetry (PIV). Such visualization studies permit a detailed explanation of the structure of the cooling curves. Results from a computational study of the free convective flow, which confirm the experimental results, are also presented.

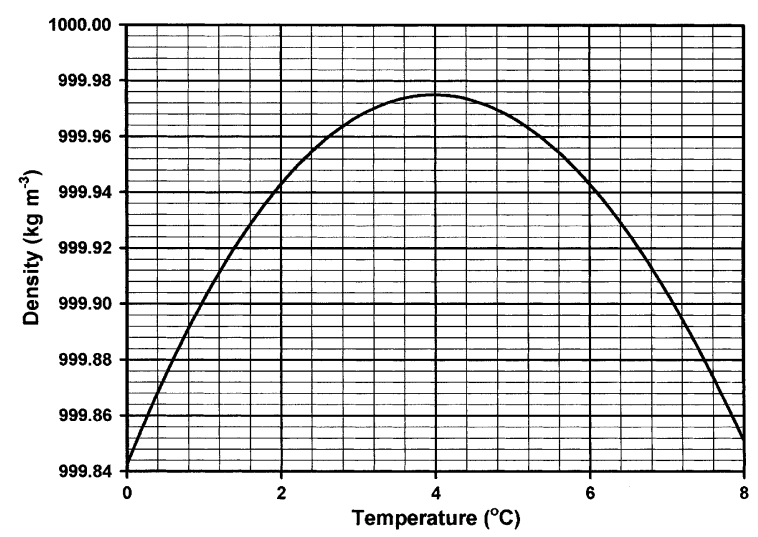

Fig. 1. Density profile of pure water at $101 \mathrm{kPa}$ in the vicinity of the density maximum.

\section{Experimental apparatus and procedure}

A schematic diagram of the apparatus used in this study is shown in Fig. 2(a), with details of the experimental chamber shown in Fig. 2(b). Two domestic refrigerator/freezer units are used to supply the coolant; one unit provides coolant at typically $8{ }^{\circ} \mathrm{C}$ (the warmer temperature, $T_{\mathrm{h}}$ ), while the other is usually set to provide coolant at $-2{ }^{\circ} \mathrm{C}$ (the colder temperature, $T_{\mathrm{c}}$ ). A mixture consisting of $20 \%$ ethylene glycol and $80 \%$ water is used as the coolant. A series of solenoid valves (V1 to V4 in Fig. 2(a)) and pumps (P1 and P2) under computer control are used to switch over from one temperature to the other. Water, or a control fluid such as ethanol, is placed in a vertical glass cylinder of inner diameter $51 \pm 1 \mathrm{~mm}$ and height $128 \pm 1 \mathrm{~mm}$ (the axis of symmetry of the cylinder is aligned to within $0.02 \mathrm{rad}$ of the vertical). The cylinder is filled with the fluid and the top is closed. This cylinder is enclosed within another glass cylinder of diameter $105 \mathrm{~mm}$, and fluid at a fixed temperature (the coolant) is pumped continuously through the annular region between the cylinders. Three thermistors (Betatherm, $2.5 \mathrm{~mm}$ diameter insulated sensor head, resistance of $5 \mathrm{k} \Omega$ at $25^{\circ} \mathrm{C}$ ) are mounted along the central axis of the inner glass cylinder, at heights of 32, 64 , and $96 \mathrm{~mm}$, to record the cooling curves (thermistor centers are positioned to $\pm 1 \mathrm{~mm}$, and temperature measurements are accurate to $0.1{ }^{\circ} \mathrm{C}$ ). Apart from the glass construction, the apparatus is thus similar to that used by Anselmi et al. [3]. The concentric glass chambers and associated plumbing are mounted in the refrigerator compartment of one of the refrigerator/freezer units, and a double-glazed window is installed in the refrigerator door to permit imaging of the convective flows.

PIV is used to visualize the flow and provide quantitative information about the velocity field. This is a technique whereby the velocity field may be inferred from the motion of tracer particles in the fluid. The water in the test chamber is seeded with polyamid spheres of 
(a)

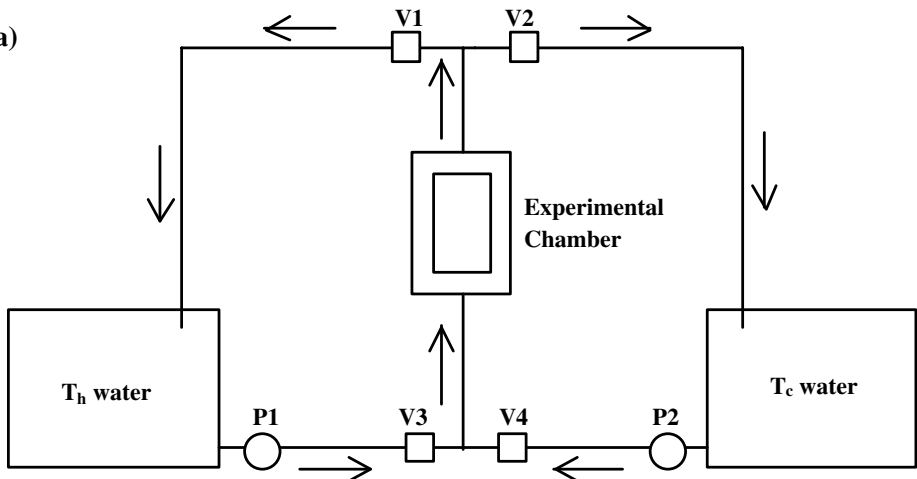

(b)

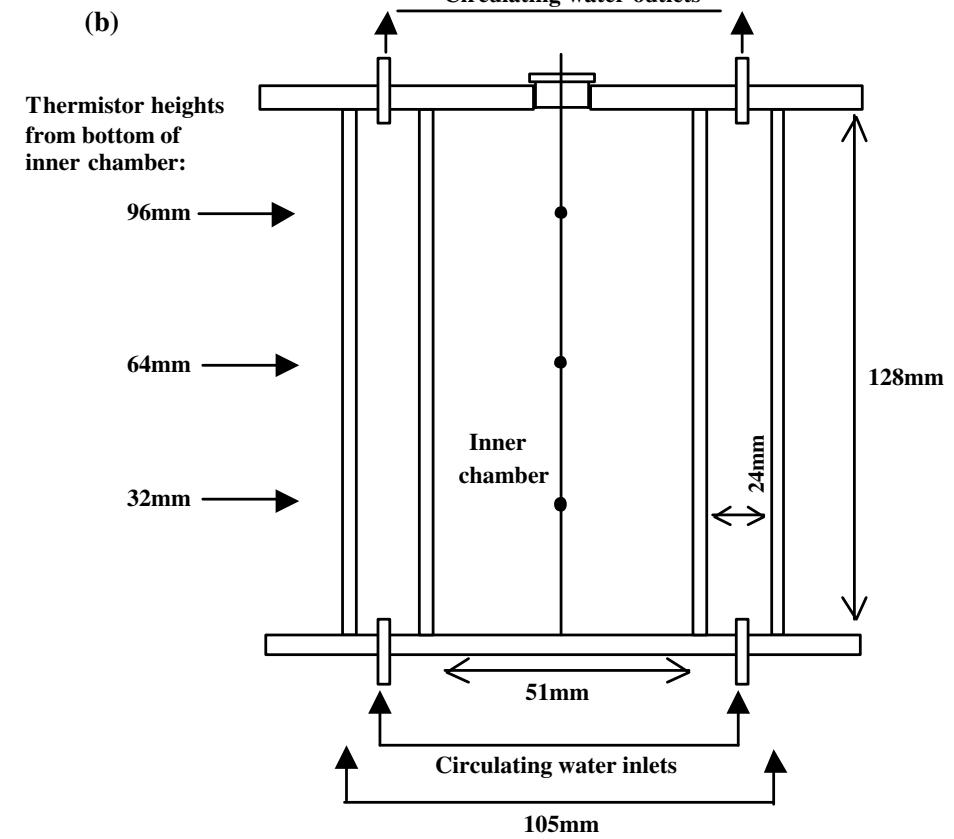

Fig. 2. (a) Overview of apparatus-V1 to V4 represent solenoid valves under computer control, and P1 and P2 are pumps, (b) schematic diagram of the experimental chamber indicating positions of the temperature probes.

$50 \mu \mathrm{m}$ diameter and density $1.03 \mathrm{~g} \mathrm{~cm}^{-3}$ (Dantec Dynamics, part number PSP-50). A collimated sheet of white light is used to illuminate a vertical plane intersecting the central axis of the concentric glass cylinders. A CCD camera $(768 \times 576$ pixels resolution) views the apparatus through the window at right angles to the illuminated plane. During the data acquisition, a PC records a sequence of images of the tracer particles. Each image is subsequently divided into interrogation windows of $32 \times 32$ pixels. The velocity of the flow in each window is calculated using cross-correlations between corresponding windows in successive images. To increase the resolution of the final vector plots the $32 \times 32$ pixel windows overlap on a grid spacing of $16 \times 16$ pixels.
The procedure used during a typical data acquisition sequence is as follows. Coolant at $8{ }^{\circ} \mathrm{C}$ is circulated through the annulus for a relatively long duration (at least one hour) so that the test fluid in the inner glass chamber reaches equilibrium at that temperature. The temperature is recorded continuously at set points along the central axis of the test chamber, and the temperature of the coolant in the annulus is also monitored. Shortly before the coolant is exchanged, the PIV particles are dispersed throughout the test fluid by blowing gas bubbles through a thin pipe inserted into the experimental chamber - the bulk flows induced by this disturbance can be seen to attenuate quickly (approx. $30 \mathrm{~s}$ ), whereas the PIV particles remain in suspension for tens 


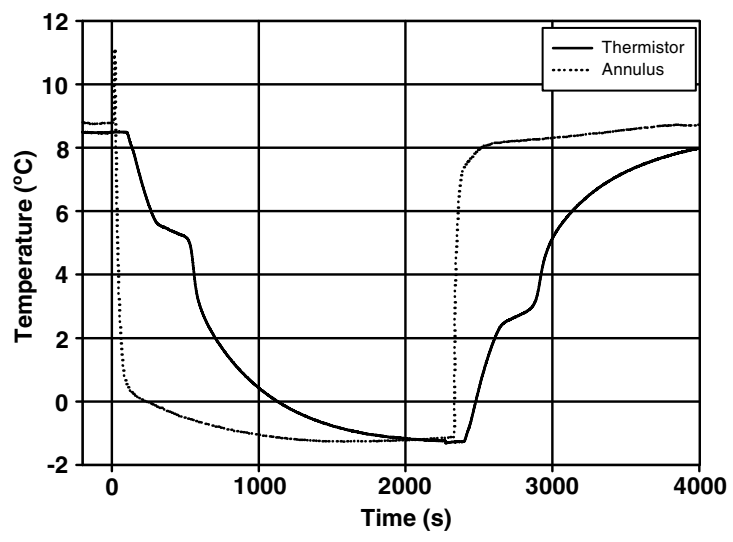

Fig. 3. Typical cooling and warming curves for water, showing the switching times for the circulation of the coolant within the annulus. The temperature probe was at the centre of the cylinder (height $=64 \mathrm{~mm}$ ).

of minutes even in the absence of convective flows (this simple agitation method obviates the need to access the chamber directly, which would result in condensation forming on the outer glass surface to the detriment of PIV image quality). To initiate convection, the $8{ }^{\circ} \mathrm{C}$ coolant is replaced by the $-2{ }^{\circ} \mathrm{C}$ coolant; the switch-over within the annulus takes of order $60 \mathrm{~s}$ to complete (Fig. 3) but there is no reason to believe that faster switching times between coolant temperatures would have major consequences for any of the results and conclusions discussed below. CCD images are recorded at 1-s intervals, and temperature readings in the experimental chamber and annulus are recorded for each image. After typically $1000 \mathrm{~s}$ (Fig. 3), the temperatures of the probes within the liquid sample have reached approximately 0 ${ }^{\circ} \mathrm{C}$, and the cooling sequence is reversed: the $-2{ }^{\circ} \mathrm{C}$ coolant is replaced by the $+8^{\circ} \mathrm{C}$ coolant and a 'warming' sequence is thus initiated. Temperature and image recording continue until the sample is back at $8{ }^{\circ} \mathrm{C}$.

\section{Experimental results}

\subsection{Structure of the cooling curves}

Fig. 4 shows a comparison between experimental cooling curves for both ethanol and water over the temperature range 8 to $0{ }^{\circ} \mathrm{C}$, for the central probe at height $64 \mathrm{~mm}$. The curve for ethanol is approximately exponential in form, indicating that the rate of heat loss is dominated by the temperature gradient between reservoir (annulus temperature) and sample - as the sample temperature decreases, the gradient declines and the rate of heat loss falls. The cooling curve for water shows the same exponential structure at the start of the cooling sequence. Flow visualization (Section 3.2) reveals a

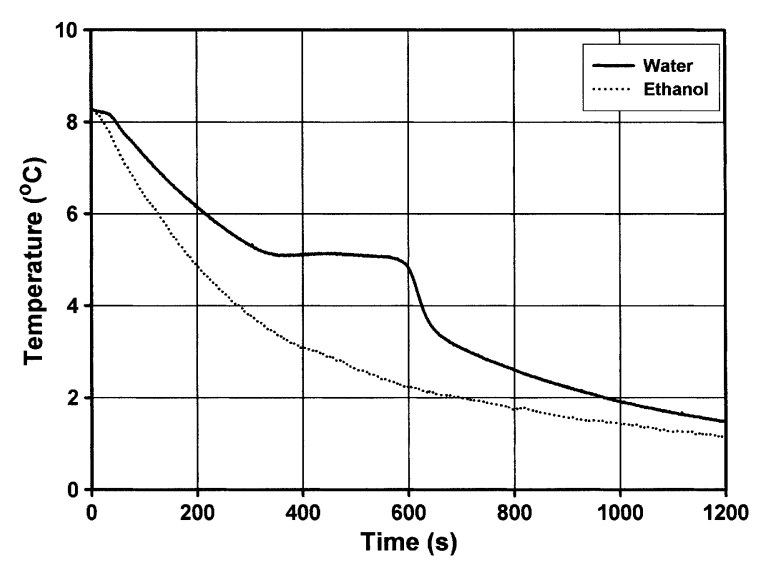

Fig. 4. Experimental cooling curves for ethanol and water, showing the plateau feature in the latter which arises due to the density maximum; temperature probe at centre of cylinder (height $=64 \mathrm{~mm}$ ).

simple convective pattern during this exponential cooling phase: fluid falls near the boundary walls due to buoyancy and this drives an upwelling of fluid in the central region of the cylinder. For ethanol, this convective cycle persists until all of the fluid reaches $T_{\mathrm{c}}$. For water, however, there is a departure from the exponential shape underlying the cooling curve. There appears an additional feature - a plateau region or 'arrest' in the temperature decline - which arises as a consequence of the non-linear density function of water (this feature is in fact evident in a graphical representation of Hope's original data - see Fig. 2 of Greenslade [6]). The onset and termination of the plateau, and the temperature at which it occurs, depend on a number of factors: the geometry of the container, the position of the temperature probe, and the temperature gradient between test sample and annulus. Fig. 5 shows cooling curves for the three probe positions along the central axis of the cylinder. It is evident that the plateau temperature increases and the width of the plateau decreases as the probe height increases. All of these effects may be understood once the cooling curve features are correlated with PIV images.

\subsection{Flow visualization}

A series of PIV quiver plots corresponding to a typical water cooling curve which brackets the density maximum temperature is shown in Fig. 6. Fig. 6(a) shows the cooling curve structure and indicates the times for which PIV plots are presented in Fig. 6(b)-(g). Fig. 6(b) shows the convective pattern at the onset of cooling: cold, dense water near the boundary wall falls, causing fluid to be forced upwards in the central region of the chamber, giving rise to a single toroidal convection cell in three dimensions. In fluids with linear density profiles, this 

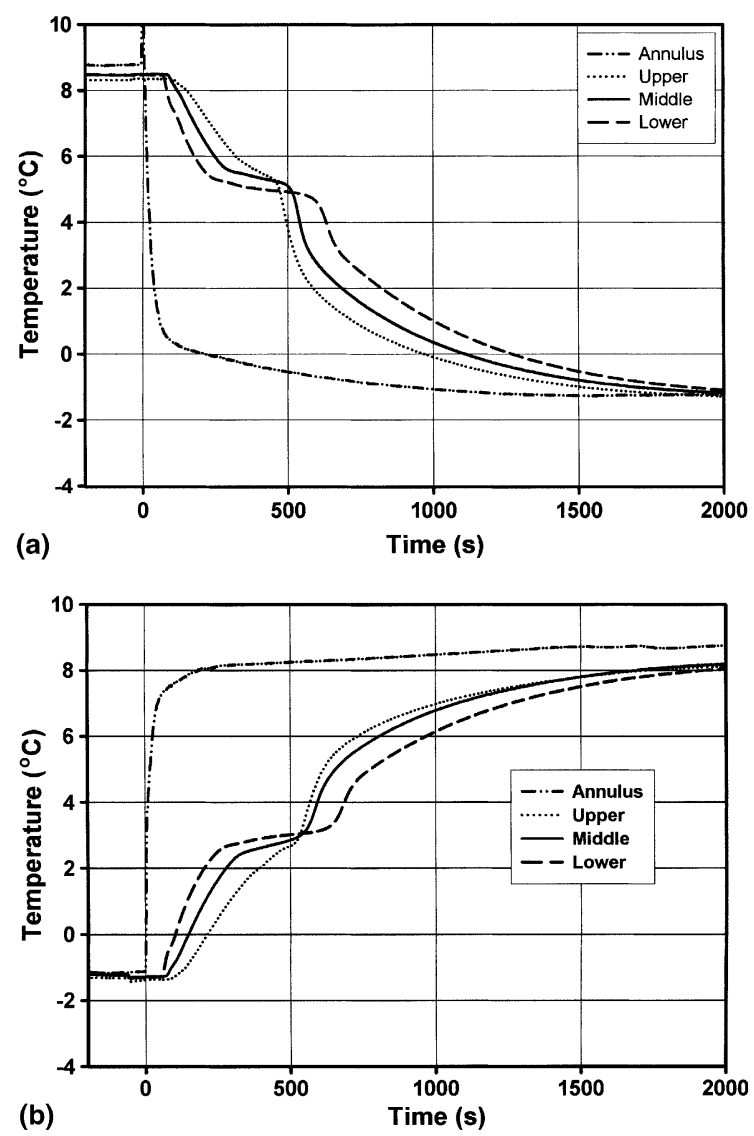

Fig. 5. (a) Cooling curves for three temperature probe positions along the vertical central axis (heights: lower $=32 \mathrm{~mm}$, mid$\mathrm{dle}=64 \mathrm{~mm}$, upper $=96 \mathrm{~mm}$ ). The annulus temperature is also shown, (b) corresponding warming curves for same probe positions.

convective structure would persist until the entire fluid reaches thermal equilibrium. In fluids where the cooling passes through a density maximum, this pattern must be disrupted in some manner, as we expect the flow direction in the torus to be reversed by the time the cooled fluid approaches equilibrium (Fig. 6(g)). The mechanism whereby this flow reversal is achieved is shown in Fig. 6(c)-(f). As the cooling progresses, some of the most dense $\left(4{ }^{\circ} \mathrm{C}\right)$ water accumulates in the lower corners. As this water is cooled below $4{ }^{\circ} \mathrm{C}$ at the lower walls, it becomes less dense and starts to rise (along the walls). This causes the descending layers of water to move inward on both sides of the section of the cylinder. The net result of these flows is to generate a vortex of water, rising along the walls on either side of the section. In the threedimensional cylinder, this would correspond to a rising sub-torus, twisting in the opposite direction to the main convective torus. One consequence of the formation and evolution of this rising vortex is the diversion of convective flow from the inner region of the cylinder; the central upwelling initiated and subsequently fed by the descending outer layers of fluid (Fig. 6(b)) is no longer sustained once the vortex develops. A temperature probe placed in this central region will detect a decline in the rate of convectively-induced cooling, as the probe is now effectively surrounded by stationary fluid (Fig. 6(d) and (e)). Thus the formation of the rising vortex accounts for the plateau in the cooling curve. Lower probes will enter the plateau earlier, as they will be the first to experience the reduction in the central upwelling. Conversely, higher probes will be the first to emerge from the plateau; this occurs when the vortex reaches the top of the cylinder and effectively spills over into the central region, inducing a descending flow of fluid in the centre of the cylinder (Fig. 6(e) and (f)). Thus, the highest probe will be the last to enter a plateau, and the first to exit from the plateau, as is evident in Fig. 5(a).

It follows from the above description that a complete reversal of the main toroidal cell flow will be achieved once the rising vortex has reached the top of the cylinder and folded over into the central region to form a descending column of liquid. The transition from Fig. 6(b)-(g) is then complete and gradient-dominated convective cooling resumes. Finally we note in this section that PIV observations of the cooling and warming sequences appear identical: at the start of both sequences, the water close to the wall descends, forcing an upwelling in the central region. At the end of each sequence, the direction of the toroidal flow is reversed, and in both sequences the vortex forms at the bottom of the cylinder and is seen to rise. This symmetry of appearance is a consequence of the symmetry of the density curve about the point of density maximum (Fig. 1) - in each case, at the start of either cooling or warming, the outer layers of water become more dense as the temperature approaches $4{ }^{\circ} \mathrm{C}$ (from above for the cooling sequence, and from below for the warming sequence).

\subsection{Plateau structure and plateau temperature as a function of probe height}

It is evident from Fig. 5(a) that the temperature at which a plateau occurs varies as a function of probe height in the column of water. During a cooling sequence, the lower probes plateau at lower temperatures. This is again consistent with the diversion of convective flow from the central region once the rising vortex has formed; at that point in time, there will be a temperature gradient in the inner region of the cylinder due to upwelling of cold water convected from the outer walls, with coldest temperatures at the bottom. This gradient is preserved when the inner flow is quenched, and remains until the vortex spills over the top of the column to resume flow in the inner region (in a reversed direction). Similarly, the plateau temperature can be seen to vary as a function of probe height during a warming sequence, 


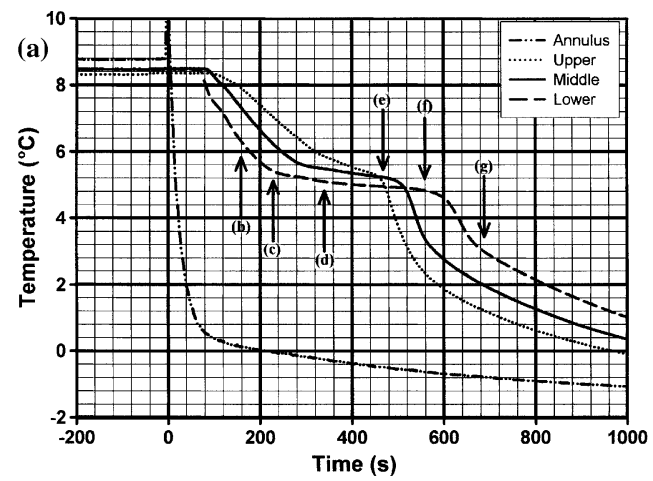

(b)

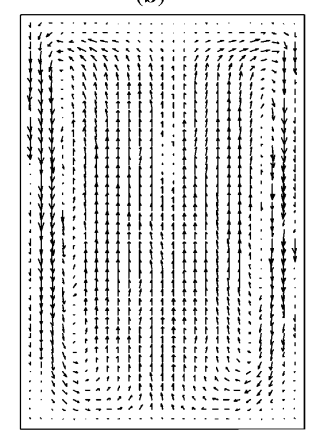

(e)

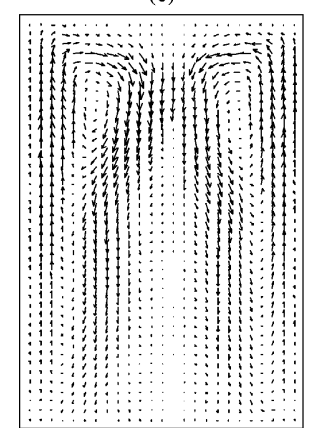

(c)

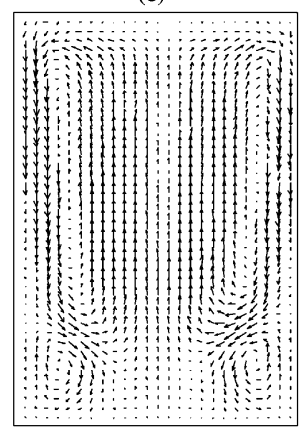

(f)

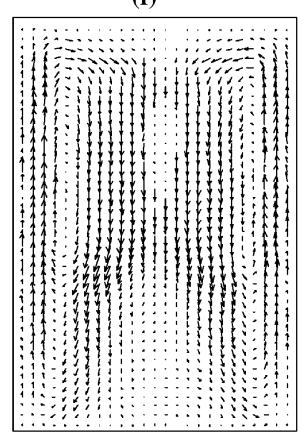

(d)

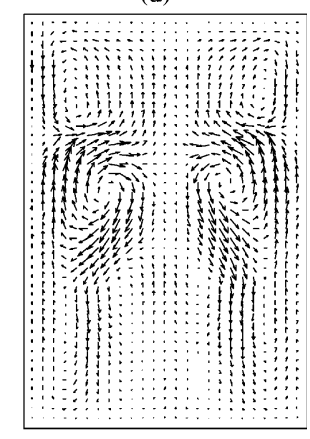

(g)

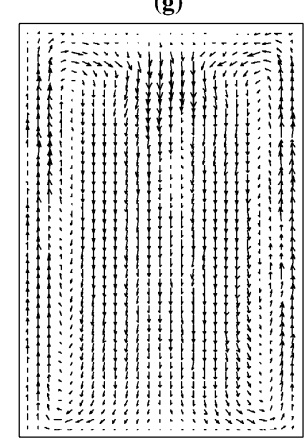

Fig. 6. (a) Cooling curves for pure water, indicating times (vertical arrows) for the PIV plots in (b) through (g). Temperature probe heights: 32, 64, and $96 \mathrm{~mm}$ for the lower, middle and upper probes, respectively, (b)-(g) velocity quiver plots obtained from PIV analysis for pure water cooling through the density maximum region.

when the water column begins at $T_{\mathrm{c}}$ and is surrounded by fluid in the annulus at the higher temperature, $T_{\mathrm{h}}$. In this case, however, the order of plateau temperatures is reversed, with the lowest probe having the highest plateau temperature (Fig. 5(b)). This occurs because water warming towards $4{ }^{\circ} \mathrm{C}$ convects in a manner identical to water cooling towards $4{ }^{\circ} \mathrm{C}$; in both cases, the layer of water at the walls descends, causing initial upwelling in the central region of the water column. During the warming sequence, when the vortex forms and cuts off this upwelling, the lowest probe will be surrounded by warmer, denser, water compared to the probes higher up on the central axis.

\subsection{Comparison of the temperatures of the cooling and warming plateaux}

A typical experimental run consists of a cooling sequence from 8 to $0{ }^{\circ} \mathrm{C}$ following by a warming sequence between 0 and $8{ }^{\circ} \mathrm{C}$. It is evident from Fig. 5 that the plateau temperature for a probe in the centre of the water column is different for the cooling and warming sequences, and that neither temperature corresponds to the temperature of maximum density. The plateau temperature for cooling $\left(T_{\mathrm{p}, \mathrm{c}}\right)$ is typically $5^{\circ} \mathrm{C}$ whereas the plateau temperature for warming $\left(T_{\mathrm{p}, \mathrm{w}}\right)$ is typically $3{ }^{\circ} \mathrm{C}$. This asymmetry between cooling and warming 
may also be explained in terms of the rising vortex. On cooling, the water close to the wall of the cylinder will cool more rapidly than water close to the centre. Thus, the rising vortex - requiring temperatures below $4{ }^{\circ} \mathrm{C}-$ will form before water in the inner region reaches $4{ }^{\circ} \mathrm{C}$, and once the vortex forms the inner region will plateau at this higher temperature. When the warming sequence is initiated, by replacing the $-2{ }^{\circ} \mathrm{C}$ water with $8{ }^{\circ} \mathrm{C}$ water in the annulus, the cold water close to the wall in the cylinder will initially descend as it heats up (as it is in the anomalous temperature region between 0 and $4{ }^{\circ} \mathrm{C}$ ). Once again a vortex will form, but in this case when the outer water regions warm to temperatures above $4{ }^{\circ} \mathrm{C}$. Water in the inner region will lag behind in temperature, and will therefore plateau below $4{ }^{\circ} \mathrm{C}$ when the vortex forms.

\section{Numerical modelling of the convective flow}

\subsection{Flow and governing equations}

To complement the experimental observations discussed in Section 3, we have carried out a series of simulations using a modified version of a two-dimensional computational fluid dynamics (CFD) algorithm. The package chosen was NaSt2D [7], an open-source CFD algorithm available in the public domain. For a specified 2-D region, and specified boundary conditions, the algorithm predicts flow patterns of a Newtonian, incompressible fluid by solving the Navier-Stokes, continuity, and Fourier (energy) equations:

$$
\begin{aligned}
& \rho_{0} \frac{\partial \mathbf{v}}{\partial t}+\rho_{0}(\mathbf{v} \cdot \nabla) \mathbf{v}=-\nabla p+\rho(T) \mathbf{g}+\mu \nabla^{2} \mathbf{v} \\
& \nabla \cdot \mathbf{v}=0 \\
& \frac{\partial T}{\partial t}+\mathbf{v} \cdot \nabla T=\alpha \nabla^{2} T
\end{aligned}
$$

where parameters were assigned the following values (appropriate for pure water at $4{ }^{\circ} \mathrm{C}[1]$ ):

$$
\begin{aligned}
& \mu=1.567 \times 10^{-3} \mathrm{~kg} \mathrm{~m}^{-1} \mathrm{~s}^{-1} \\
& \alpha=1.344 \times 10^{-7} \mathrm{~m}^{2} \mathrm{~s}^{-1} \\
& \rho_{0}=999.9720 \mathrm{~kg} \mathrm{~m}^{-3} \\
& \text { and } g=9.81 \mathrm{~m} \mathrm{~s}^{-2} .
\end{aligned}
$$

The Boussinesq approximation is assumed, whereby the temperature-dependence of the density is only considered in the buoyancy term of the Navier-Stokes equation [8].

The state equation used in NaSt2D assumes a linear density response as a function of temperature:

$\rho=\rho_{0}\left(1-\beta\left(T-T_{0}\right)\right)$.

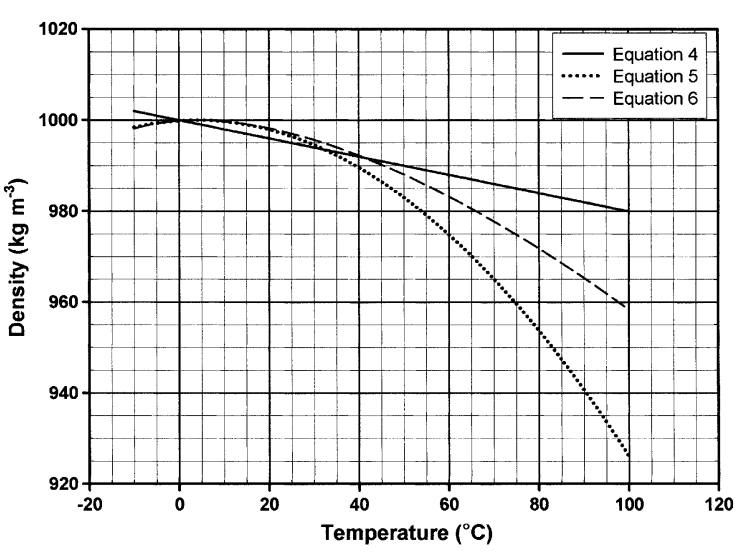

Fig. 7. The three state functions used in the CFD simulations.

Values used in the simulations of water exhibiting a linear temperature response were $T_{0}=0.0{ }^{\circ} \mathrm{C}$, $\rho_{0}=999.972 \mathrm{~kg} \mathrm{~m}^{-3}$, and $\beta=2.0 \times 10^{-4} \mathrm{~K}^{-1}$. In addition to this linear state function, two non-linear state equations were tested:

$\rho=c_{0}+c_{1} T+c_{2} T^{2}$

where $c_{0}=999.845079, c_{1}=0.06378, c_{2}=-0.0080125$ (with $\rho$ in $\mathrm{kg} \mathrm{m}^{-3}$ ), and

$\rho=\left(c_{0}+c_{1} T+c_{2} T^{2}+c_{3} T^{3}+c_{4} T^{4}+c_{5} T^{5}\right) /\left(1+d_{1} T\right)$

where $c_{0}=999.83952, c_{1}=16.945176, c_{2}=-7.9870401 \times$ $10^{-3}, \quad c_{3}=-46.170461 \times 10^{-6}, \quad c_{4}=105.56302 \times 10^{-9}$, $c_{5}=-280.54253 \times 10^{-12}$, and $d_{1}=16.87985 \times 10^{-3}$.

State function (5) is symmetric about the density maximum, based on a second-order polynomial fit to data taken from [1]; state function (6), taken directly from [1], is asymmetric about the density maximum, which occurs at $3.98{ }^{\circ} \mathrm{C}$ (a more recent edition of the Handbook of Chemistry and Physics [9] presents slightly different data for the density of water as a function of temperature, and the higher order asymmetrical polynomial fit is omitted). Graphs of the three equations of state are shown in Fig. 7.

\subsection{Boundary and initial conditions}

The two-dimensional region enclosing the fluid is shown in Fig. 8. The boundary conditions were as follows:

$u=v=0$ for all walls,

$\frac{\partial T}{\partial y}=0$ on planes $y=0$ and $y=h$ (adiabatic top and bottom walls),

$T=T_{\mathrm{c}}$ (cooling sequence) or $T=T_{\mathrm{h}}$ (warming sequence) for $x=0$ and $x=w$ (isothermal side walls). 


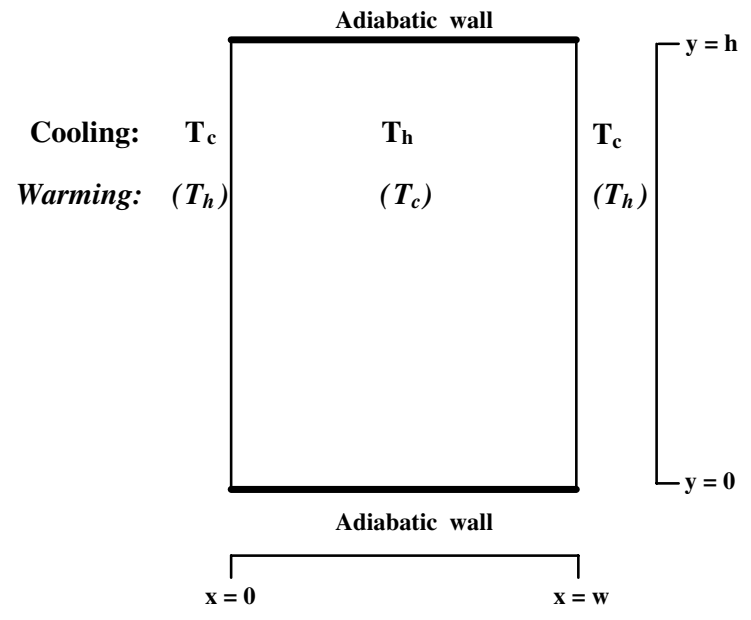

Fig. 8. Schematic view of 2D cavity used in CFD model.

Initially, the fluid is at $T_{\mathrm{h}}$ (cooling sequence) or at $T_{\mathrm{c}}$ (warming sequence). A dimensional version of $\mathrm{NaSt} 2 \mathrm{D}$ was used for the purposes of these simulations; the following values were assigned to the size and temperature variables: $w=0.051 \mathrm{~m}, h=0.128 \mathrm{~m}, T_{\mathrm{c}}=0{ }^{\circ} \mathrm{C}, T_{\mathrm{h}}=8$ ${ }^{\circ} \mathrm{C}$. A grid of $24 \times 45$ cells (horizontal $\times$ vertical) was used, with a time-step resolution of $0.025 \mathrm{~s}$. Finer grid and time resolutions were also tested, but were not found to alter the simulation results.

\subsection{Simulation results and comparison with observations}

It is evident from Fig. 7 that the state functions (5) and (6) are closely matched over the temperature range of interest $\left(0-8{ }^{\circ} \mathrm{C}\right)$ and they yield virtually identical results for convective simulations over this temperature range. Cooling and warming curves are shown for the three state functions (4)-(6) in Fig. 9. As is to be expected, the cooling and warming curves corresponding to the linear state function (4) are approximately exponential in form, with no plateau features. The cooling and warming curves for the non-linear state functions (5) and (6) lie on top of each other, indicating that the flow behaviour over the temperature range $0-8{ }^{\circ} \mathrm{C}$ is insensitive to differences between these two functions. In particular, the plateau temperatures predicted by these two state functions are the same, and do not correspond to a temperature of $4{ }^{\circ} \mathrm{C}$ in either the cooling or warming sequence. It is evident, therefore, that the displacements of the plateau temperatures from $4{ }^{\circ} \mathrm{C}$ are not associated with the small asymmetry in the water density profile about the temperature of maximum density, contrary to the hypothesis of Sonnino and de Paz [4].

The experimental cooling curves shown in Fig. 6(a) may be compared with the CFD cooling curves of Fig.

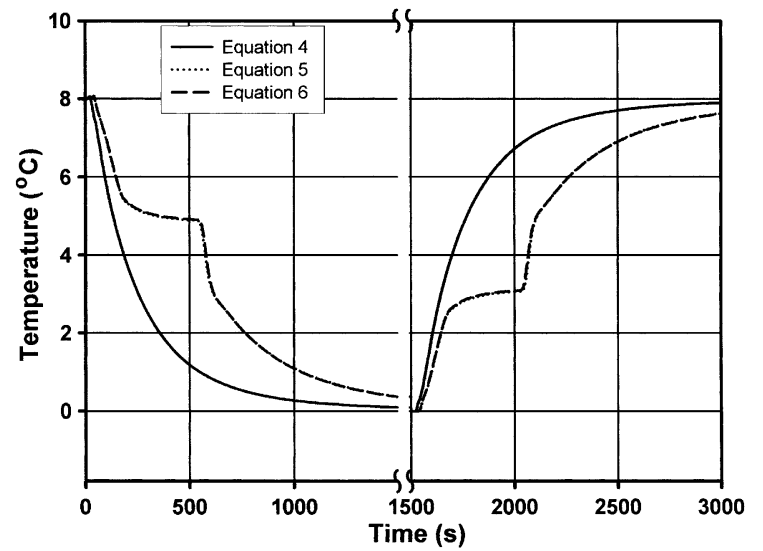

Fig. 9. Cooling and warming curves from CFD, for the three state functions shown in Fig. 7. The temperature probe position in these simulations was at the mid-point of the central vertical axis (height $=64 \mathrm{~mm}$ ). Curves for state functions 5 and 6 are almost identical, and fall in close proximity to each other on the graph. The discontinuity in the time axis indicates that the cooling curves were truncated at $t=1500 \mathrm{~s}$.

10(a). A correlation coefficient, $r$, may be calculated between variables $x$ and $y$ (experiment and CFD model) summed over $n$ points as follows [10]:

$r=\frac{\sum_{i=1}^{n}\left(x_{i}-\bar{x}\right)\left(y_{i}-\bar{y}\right)}{\left[\sum_{i=1}^{n}\left(x_{i}-\bar{x}\right)^{2} \sum_{i=1}^{n}\left(y_{i}-\bar{y}\right)^{2}\right]^{1 / 2}}$

where $r$ may assume values between 1.0 (perfect correlation) and -1.0 (anticorrelation). Correlation coefficient values of $0.988,0.996$, and 0.991 (for 30 points) are found when a comparison is made between the upper, middle, and lower temperature probes, respectively. A similar 30-point comparison between the water and ethanol cooling curves in Fig. 4 yields a correlation coefficient of 0.930 . All of these coefficient values are close to unity, indicating that the curves correlate well in each case. It may however be inferred from the changes in the values that the central probe profiles are in best agreement, while - as expected - the water and ethanol curves of Fig. 4 result in the poorest agreement. Differences which are evident between the cooling curves of Figs. 6(a) and 10(a) are most likely due to the numerous approximations which are made in the CFD calculation, including the assumption of constant values for the viscosity and thermal diffusivity (independent of temperature), the assumption that the density changes only in the buoyancy term of the momentum equations, and due to the fact that the CFD model is two-dimensional, whereas the PIV results are obtained for a two-dimensional slice through a three-dimensional cylinder. In addition, the boundary conditions in the experiment will not match the ideal conditions assumed in the CFD 


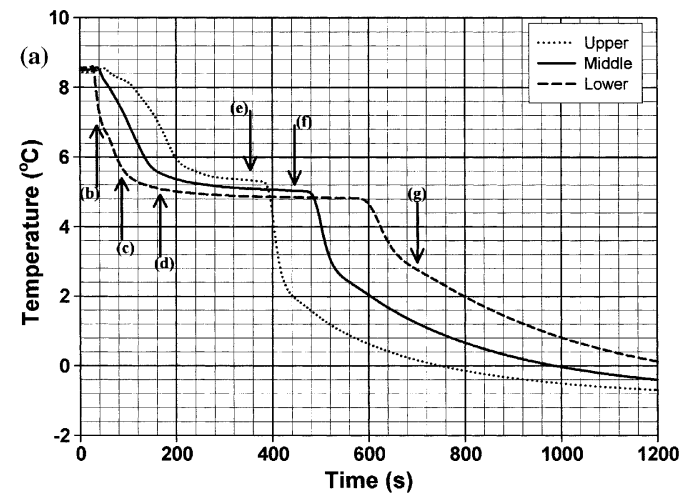

(b)

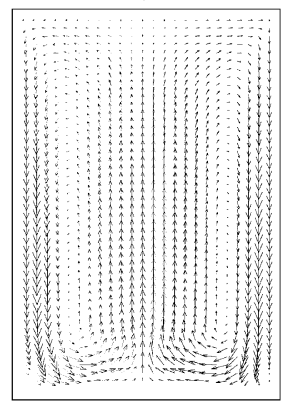

(e)

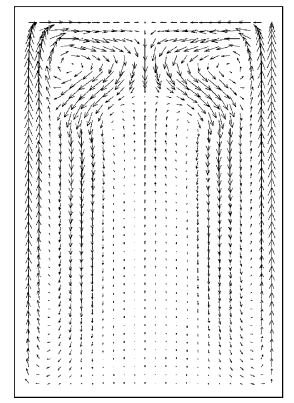

(c)

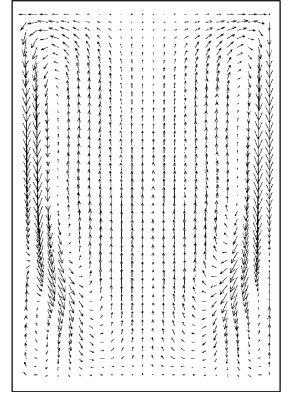

(f)

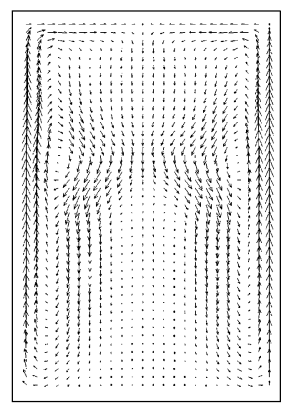

(d)

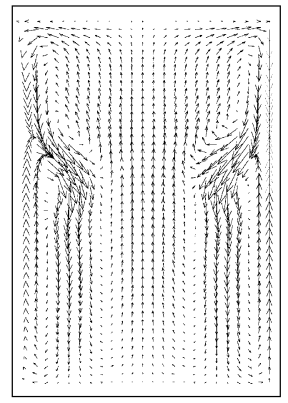

$(\mathrm{g})$

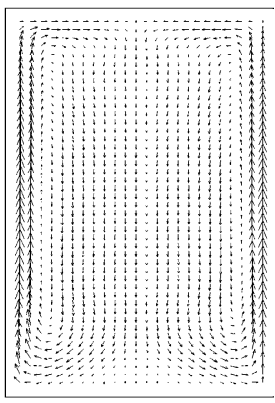

Fig. 10. (a) Cooling curves from CFD model, indicating times (vertical arrows) for the velocity quiver plots in (b) through (g). Temperature probe heights: 32, 64, and $96 \mathrm{~mm}$ for the lower, middle and upper probes, respectively, (b)-(g) velocity quiver plots from CFD showing stages of convective flow development corresponding to the equivalent plots of Fig. 6.

model. Velocity quiver plots generated by the CFD model are shown in Fig. 10(b)-(g). These may be compared with the experimental PIV plots of Fig. 6(b)-(g). The CFD plots were chosen to correspond to the same stages of development of the convective flow as shown in the PIV plots of Fig. 6. The times corresponding to these velocity plots are indicated on the cooling curves in Figs. 6(a) and 10(a); differences between these times for the experimental results and the CFD model may again be ascribed to the model approximations.

Velocity profiles for two different heights in the cavity are shown in Fig. 11 for both PIV measurements and CFD calculations. The comparison between experiment and theory is carried out for a time when the rising vortex is approximately mid-way up the cavity as shown in the velocity quiver plots in Fig. 11(a) and (b) (the time when this appears to occur in the CFD calculation does not match with experiment due to differences in the shapes of the experimental and calculated cooling curves). As with the comparison between the experimental and theoretical cooling curves, the velocity profiles are in agreement in terms of overall trends, but they do not agree in detail. The velocity components for the upper profile (line A in Fig. 11(a) and (b)) are in reasonable agreement (correlation coefficients of 0.93 and 0.95 for 24 points of comparison, for the $x$-component and $y$-component of the velocity vectors, respectively). There is less agreement, however, between the velocity 
(a)
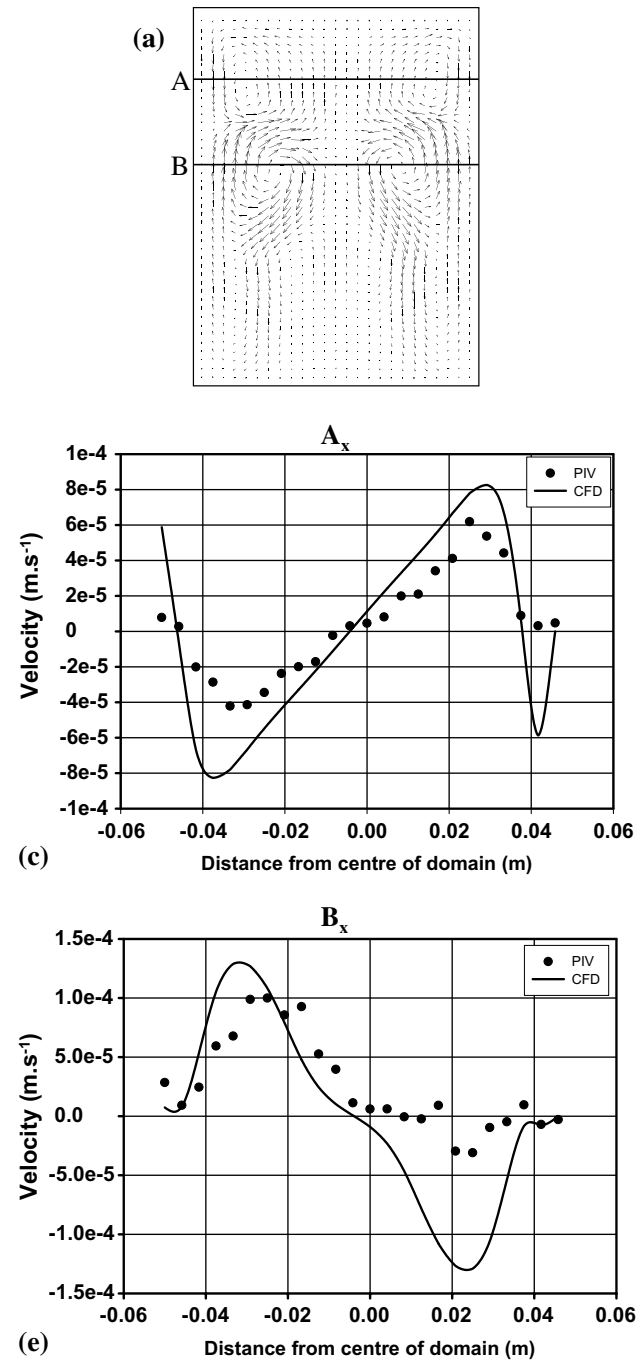

(b)

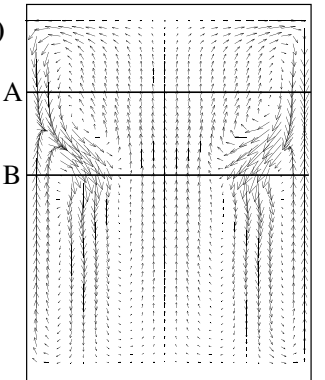

$\mathbf{A}_{\mathbf{y}}$

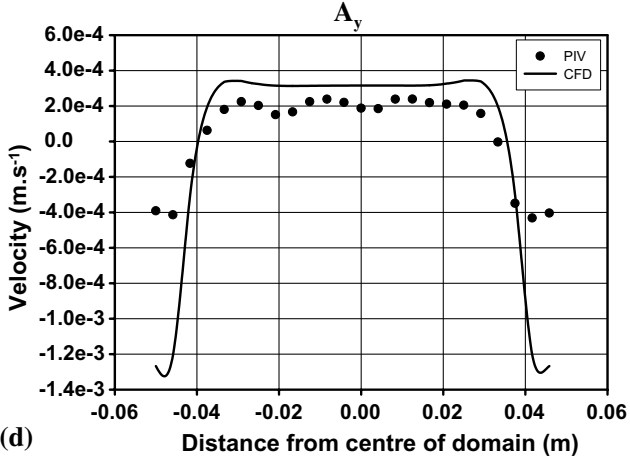

$\mathbf{B}_{\mathbf{y}}$

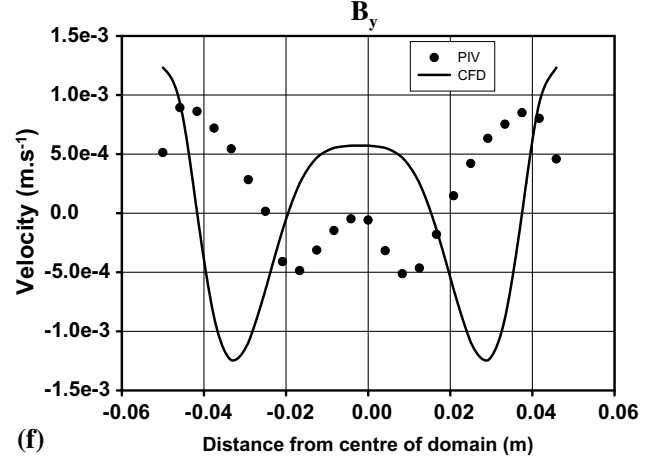

Fig. 11. (a) Velocity quiver plot from PIV analysis of experimental data at an instant when the rising vortex is mid-way up the cylinder, (b) velocity quiver plot from CFD showing the rising vortex at the mid-point, (c) profile of the $x$-component of the velocities along line $\mathrm{A}$ in plots (a) and (b), (d) profile of the $y$-component of the velocities along line A, (e) profile of the $x$-component of the velocities along line $\mathrm{B}$ in plots (a) and (b), (f) profile of the $y$-component of the velocities along line B.

profiles which cut through the vortices (line B in Fig. $11(\mathrm{a})$ and (b)): the comparison yields correlation coefficients of 0.87 and -0.19 for the $x$-component and $y$-component of the velocity vectors. Once again the discrepancies are most likely due to the approximations made in the CFD model, and to the assumption of ideal boundary conditions which are not realized in the experiment.

\section{Discussion}

The combination of flow visualization and flow simulation has facilitated a detailed description of how free convection in a vertical cylinder of water is modified in the vicinity of the fluid density maximum. Free convection in a vertical cylinder of fluid with uniform side wall cooling results in a relatively simple flow pattern if the density state function is linear: fluid close to the boundary walls falls relative to the fluid in the interior of the cylinder, and this initiates a cyclical flow whereby boundary fluid descends and interior fluid rises, and there is a continual exchange until thermal equilibrium is reached. It has been shown how this flow pattern is modified in the presence of a density maximum, such as occurs in water at $4{ }^{\circ} \mathrm{C}$ at $101 \mathrm{kPa}$. The simple cycle is disrupted when fluid at the density maximum begins to accu- 
mulate in the lower side regions of the cylinder. Less dense fluid descending above this accumulation is diverted inward, but is then entrained in a vortex formed when the fluid at maximum density cools even further, and starts to rise along the boundary walls. Two convective patterns are now present in the enclosure: the simple cyclical pattern persists in the upper regions of the cylinder, but is gradually forced to contract as the vortex rises along the boundary walls. This double pattern persists until the vortex reaches the top wall of the cylinder and initiates a downward flow in the interior. The single, simple convective cycle is thus restored, but with the flow direction reversed: fluid now rises along the boundary walls and descends in the interior. An identical sequence of events takes place if the fluid is initially at a temperature below the density maximum, and is uniformly heated via the side walls. Initially, the fluid close to the vertical walls again begins to descend, as the warmer fluid is now more dense than the cooler, interior fluid. Again, as the boundary reaches the temperature of maximum density, some fluid accumulates in the lower side regions, and then starts to rise along the walls, forming the same vortex pattern as before. Finally, when the bulk temperature is above the temperature of maximum density, the simple convective cycle is restored, with its direction reversed: warming fluid-now above the temperature of maximum density and in the normal linear region of the density state function-rises along the walls as expected, and the interior fluid descends. These modifications in the flow patterns results in a temporary flattening of the cooling and warming curves which are recorded at points along the central axis of the cylinder: the slowing of the temperature change arises due to the diversion of convective flow away from the central region by the rising vortex. The plateau is observed to occur at different temperatures depending on the height of the temperature probe along the central axis, and it is also observed that the cooling plateau temperature $\left(T_{\mathrm{p}, \mathrm{c}}\right)$ is not the same as the warming plateau temperature $\left(T_{\mathrm{p}, \mathrm{h}}\right)$. These effects arise from the formation of a vertical temperature gradient during the initial stages of convection, which is then preserved when the flow is deflected away from the central region. In the cooling sequence, the gradient is bottom (cooler) to top (warmer), whereas this gradient is reversed for a warming sequence.

The alteration of free convection due to the presence of the density anomaly has implications for a variety of areas of study. Wilson [11] exploited the reduction in convective flows in water near $4{ }^{\circ} \mathrm{C}$ to study settling velocities of microscopic glass spheres (diameters in the range $2.5-10 \mu \mathrm{m}$ ). At temperatures removed from $T_{\rho \max }$, such measurements are more difficult due to the presence of convection currents, and the degree of temperature control necessary to eliminate such currents is difficult to achieve in practice. Goren [12] has shown that for water at $4{ }^{\circ} \mathrm{C}$ the convective velocities are reduced by about 8 fold and 80 -fold from those in water at $20{ }^{\circ} \mathrm{C}$ for temperature variations of 1 and $0.01{ }^{\circ} \mathrm{C}$ respectively. Such effects arise from the slow variation of density as a function of temperature $(\mathrm{d} \rho / \mathrm{d} T)$ in the vicinity of the density maximum. On the other hand, the evidence presented in Section 3 for the formation of a rising vortex of water, starting in the lower edge regions of the cylinder, may have implications for the accumulation of sediments in such regions. It is envisaged that sediment accumulation will be disrupted or inhibited due to this vortex formation, compared to sedimentation in water over temperature ranges not encompassing $T_{\rho \max }$. Further work in this area is underway. The variation of $T_{\rho \max }$ as a function of salinity may have implications for convective currents in low-salinity regions of the oceans. Fennel [13] postulates that the variations in time of occurrence of phytoplankton spring blooms in the Baltic Sea are influenced by whether or not the winter water temperature drops below $T_{\rho \max }$, and this temperature in turn varies from location to location within the Baltic region due to salinity variations ( $T_{\rho \max }$ being typically in the range $1.6-2.2{ }^{\circ} \mathrm{C}$ ).

\section{Acknowledgements}

P.McB. gratefully acknowledges financial support from Enterprise Ireland, the state funding agency for pure research.

\section{References}

[1] Handbook of Chemistry and Physics, 65 ed., CRC Press, Boca Raton, Florida, 1984, F-5, F-37, D-175, E-10.

[2] T.C. Hope, Experiments and observations upon the contraction of water by heat at low temperatures, Trans. Royal Soc. Edinburgh 5 (1805) 379-405.

[3] C. Anselmi, M. de Paz, A. Marciano, M. Pilo, G. Sonnino, Free convection experiments in water and deuterated mixtures at temperatures including the density maxima, Int. J. Heat Mass Transfer 33 (11) (1990) 2519-2524.

[4] M. De Paz, M. Pilo, G. Sonnino, Non-linear, unsteady free convection in a vertical cylinder submitted to a horizontal thermal gradient: measurements in water between 6 and 21 ${ }^{\circ} \mathrm{C}$ and a theoretical model of convection, Int. J. Heat Mass Transfer 30 (2) (1987) 289-295.

[5] G. Sonnino, M. De Paz, Comparison between experimental data and theoretical calculations of free convection in water near its density maximum, Math. Comput. Model. 25 (6) (1997) 107-115.

[6] T.B. Greenslade, The maximum density of water, Phys. Teach. 23 (1985) 474-477.

[7] M. Griebel, T. Dornseifer, T. Neunhoeffer, Numerical Simulation in Fluid Dynamics-A Practical Introduction, 
Society for Industrial and Applied Mathematics (SIAM), Philadelphia, 1998 (Chapters 2, 3, 9).

[8] D.J. Tritton, Physical Fluid Dynamics, second ed., Clarendon Press, Oxford, 1988 (Chapter 14).

[9] Handbook of Chemistry and Physics, 83rd ed., CRC Press, Boca Raton, Florida, 2002, section 6, p. 5.

[10] J.R. Taylor, An Introduction to Error Analysis, University Science Books, California, 1982.
[11] B.W. Wilson, The sedimentation of dilute suspensions of microscopic spheres in water, Aust. J. Appl. Sci. 3 (1952) 252-257.

[12] S.L. Goren, On free convection in water at $4{ }^{\circ} \mathrm{C}$, Chem. Eng. Sci. 21 (1966) 515-518.

[13] K. Fennel, Convection and the timing of phytoplankton spring blooms in the western Baltic Sea, Estuar. Coast. Shelf Sci. 49 (1999) 113-128. 